\section{Postnatal transitional weight loss and adverse outcomes in extremely premature neonates}

\author{
Rita P. Verma,1 Syed Shibli, ${ }^{2}$ \\ Eugene Komaroff ${ }^{3}$
}

1Department of Pediatrics, Nassau University Medical Center, East Meadow, NY; '2LIJ Health System, Manhasset, NY; ${ }^{3}$ Keiser University Graduate School, Fort Lauderdale, FL, USA

\section{Abstract}

The early postnatal weight loss (EPWL) is highly variable in the extremely low birth weight infants (birth weight $<1000 \mathrm{~g}$, ELBW). It is reported to be unassociated with adverse outcomes within a range of 3$21 \%$ of birth weight. Its wide range might have contributed to this lack of association. The aim of our paper is to study the effects of maximum EPWL, graded as low, medium and large on clinical outcomes in ELBW infants. In a retrospective cohort observational study EPWL was measured as maximum weight loss from birth weight (MWL) in ELBW infants and grouped as low (5-12\%) moderate (18.1-12\%) and high $(18-25 \%)$. The clinical course and complications of infants were compared between the groups. Gestational age (GA) was highest and surfactant administration, peak inspiratory pressure requirement, fluid intake, urinary output, oxygen dependent days and the number of oxygen dependent infants at age 28 days were lower in the low MWL compared to the high MWL group. However, all these significant P-values declined after controlling for GA. Diabetes mellitus and pregnancy associated hypertension were not noted in mothers in high MWL group, whereas $38 \%$ of mothers in low MWL group suffered from the latter $(\mathrm{P}=0.05)$. Maximum postnatal transitional weight loss, assessed in the range of low, moderate and high, is not associated with adverse outcomes independent of gestational age in ELBW infants. Maternal hypertension decreases EPWL in them.

\section{Introduction}

Early postnatal weight loss (EPWL) is an important physiological component of normal transition to extra uterine life. ${ }^{1-4}$ It represents the postnatal contraction of an expanded fetal extracellular water volume
(ECW). ${ }^{2}$ Whereas, the abnormal postnatal persistence of high intra uterine ECW has been associated with significant morbidities in premature neonates, EPWL in the wide range of $3-21 \%$ is not independently correlated with major diseases attributed to abnormal body water metabolism in extremely low birth weight infants (ELBW, birth weight $<1000 \mathrm{~g}$ ), the population which is most susceptible to such disorders. ${ }^{4} \mathrm{We}$ speculated that the documented wide range and unpredictable variability in EPWL might have contributed to this lack of association. Earlier studies, which correlated lower EPWL to BPD or death, did not specify the method utilized for calculation of weight loss.., 6 In this study we evaluated the association of maximum EPWL, graded as low, medium and high, with morbidities which are related to water metabolism in ELBW infants. The goal was to identify if a particular range of EPWL will be associated with adverse outcomes, and therefore, should be avoided in this highly vulnerable group of premature infants.

\section{Materials and Methods}

In an observational cohort study, prospectively entered data were retrospectively extracted and early postnatal maximum weight loss (MWL) from birth weight was calculated in ELBW infants. The relationship of MWL with clinical conditions associated with abnormal body fluids metabolism was analyzed under three groups according to the volume: low (5.0$12 \%)$, moderate (12.1-18\%), and high (18.1-25\%). Our previously described population and methodologies, as briefly outlined below were utilized for the study. ${ }^{4}$ The protocol was approved by the institutional review board.

\section{Determination of body weight}

The infants were weighed daily at 6:00 AM by the nursing staff on a Scale-tronix 4800 scale (Scale-tronix, Inc. Wheaton, IL, USA). Attached sensors, tubes and lines were held by the nurses to achieve the most accurate measurement. Body weight was not corrected to a possible remaining error as the error was assumed to be in average the same every day and therefore not relevant.

\section{Environment}

All infants were kept either in an open warmer bed (Air shields Vickers, Hatboro, PA, USA) or in an incubator (Air shields Isolette C 2000 Hatboro, PA, USA) in a neutral thermal environment and at times in
Correspondence: Rita P. Verma, Department of Pediatrics, Nassau University Medical Center, 2201 Hempstead Turnpike, East Meadow, NY 11554, USA.

Tel.: +1.6318755375 .

E-mail: ritaverma@aol.com

Key words: extremely preterm neonates; postnatal weight loss; neonatal morbidities; extra uterine transition; neonatal outcomes.

Contributions: RPV conceptualized and wrote the protocol, as well as the article; SS collected data and EK analyzed them.

Conflict of interest: the authors declare no potential conflict of interest.

Received for publication: 11 October 2016. Revision received: 6 March 2017.

Accepted for publication: 22 March 2017.

This work is licensed under a Creative Commons Attribution NonCommercial 4.0 License (CC BY-NC 4.0).

C Copyright R.P. Verma et al., 2017

Licensee PAGEPress, Italy

Pediatric Reports 2017; 9:6962

doi:10.4081/pr.2017.6962

relatively high $(>70 \%)$ humidity as per the attending neonatologist's decision. Infants were started on phototherapy if the indirect serum bilirubin exceeded the level of 5 $\mathrm{mg} / \mathrm{dL}$.

\section{Fluids, electrolyte and nutrition}

Fluid, electrolytes and nutritional management was determined by the attending neonatologists as per their assessment. Parenteral fluids were started via umbilical or peripheral catheters on admission and parenteral nutrition on day of life 1 . The volume of total fluids given was 80 to 120 $\mathrm{mL} / \mathrm{kg}$ /day on DOL 0 and thereafter adjusted by the attending neonatologist according to the body weight, serum sodium levels, and evolving complications. The enteral nutrition was started as soon as clinically permissible.

\section{Statistical analysis}

Student's t, Chi Square and Fisher's exact tests were utilized for group comparisons. A multivariate analysis was performed to control for confounding variables. The probability $(\mathrm{P})$ value was set at 0.05 for significance. The SAS statistical system (SAS Institute Inc., Cary, NC, USA) was used for the study. 


\section{Results}

A total of 102 eligible infants were born during the designated study period, out of which 11 were excluded for MWL exceeding $25 \%$ (8) or being $<5 \%$ (3). A total of 91 infants were included in the final analysis.

The mean and standard deviation of MWL in all 82 infants was 15.03 and $4.3 \%$. Gestational age (GA) was higher in the low MWL group compared to the rest. It did not differ between the moderate and high groups (Table 1). The receipt of surfactant; day of life (DOL) of regaining birth weight (BW); mean maximum PIP during DOL 1$7(\mathrm{P}=0.06)$, fluid volume intake and urinary output during the first week of life; total days on oxygen and the number of infants who were oxygen dependent on DOL 28 were higher in the high MWL group com- pared to the low group (Tables 1 and 2). After controlling for GA, all these comparisons lost significance, except for the DOL on which birth weight was regained $(\mathrm{P}<0.05)$. All infants in the three groups were subjected to phototherapy for hyperbilirubinemia during the first 10 days of life. When compared with the moderate MWL group, the infants in low group had higher GA $(\mathrm{P}=0.01)$ and they remained on oxygen for less number of days $(\mathrm{P}=0.06)$. Moderate and high MWL groups differed only in terms of days of life of MWL and BW regained, which were both higher in the latter group. Complications, such as, diabetes mellitus or pregnancy associated hypertension (PAH) were not noted in mothers in high MWL group, whereas 38\% of mothers in low MWL group suffered from PAH (Table 1, $\mathrm{P}=0.05$ ).

\section{Discussion and Conclusions}

The transitional early postnatal maximum weight loss of 3 to $21 \%$ from birth weight is reported to be unassociated with morbidities in ELBW neonates. ${ }^{4}$ Our evaluation of EPWL under three discrete volume ranges produced similar results. These reports suggest that the critically ill extremely premature newborn infants might be able to modify their transitional EPWL according to the con-current morbidities and other factors that influence the body water balance, such as, par-enteral fluid supplementation, medications, ambient temperature and humidification. The results also validate the current principles of fluid management in these infants.

Early neonatal weight loss is attributed to the postnatal contraction of extracellular

Table 1. Demographic and clinical characteristics of infants and mothers in the three groups.

\begin{tabular}{|c|c|c|c|c|}
\hline Variable & Low MWL $(n=21)$ & Moderate MWL $(\mathrm{n}=52)$ & High MWL ( $n=18)$ & $\mathbf{P}$ \\
\hline Body weight (g) & $786 \pm 188$ & $784 \pm 130$ & $810 \pm 118$ & NS \\
\hline Gestational age (weeks) & $27 \pm 1$ & $26 \pm 1.5$ & $25 \pm 1.5$ & $\mathrm{a}=0.01, \mathrm{~b}=0.002$ \\
\hline C. section & $19(90)$ & $36(70)$ & $7(39)$ & $\mathrm{b}=0.05$ \\
\hline Male & $11(55)$ & $26(50)$ & $10(55)$ & NS \\
\hline Caucasian & $13(62)$ & $26(57)$ & $12(66)$ & NS \\
\hline Surfactant receipt & $13(62)$ & $40(78)$ & $18(100)$ & $\mathrm{b}=0.04$ \\
\hline Mean Maximum FiO2 during DOL 1-7 (\%) & $35 \pm 14$ & $36 \pm 10$ & $36 \pm 10$ & NS \\
\hline Mean Maximum PIP during DOL 1-7 $\left(\mathrm{cm} / \mathrm{H}_{2} \mathrm{O}\right)$ & $11 \pm 6$ & $14 \pm 6$ & $15 \pm 4$ & $\mathrm{~b}=0.06$ \\
\hline DOL of Maximum weight loss (d) & $5.6 \pm 3$ & $5 \pm 1.4$ & $6.5 \pm 2.3$ & $\mathrm{c}=0.02$ \\
\hline DOL when birth weight was regained (d) & $12 \pm 3$ & $14 \pm 3$ & $18 \pm 4$ & $\mathrm{~b}=0.01, \mathrm{c}=0.001$ \\
\hline Mean fluid intake during DOL 1-7 (mL/kg) & $140 \pm 15$ & $145 \pm 20$ & $155 \pm 20$ & $b=0.03$ \\
\hline Mean urinary output during DOL 1-7 (mL/kg) & $98 \pm 22$ & $105 \pm 27$ & $119 \pm 23$ & $b=0.02$ \\
\hline DOL when full feeds achieved (d) & $25 \pm 13$ & $23 \pm 13$ & $23 \pm 10$ & NS \\
\hline Discharge weight (g) & $2166 \pm 308$ & $2222 \pm 413$ & $2129 \pm 226$ & NS \\
\hline Maternal antibiotics & $12(57)$ & $31(60)$ & $18(100)$ & $\mathrm{b}=0.06$ \\
\hline Ante natal steroid & $21(100)$ & $43(83)$ & $15(83)$ & NS \\
\hline Chorioamnionitis & $2(11)$ & $10(19)$ & $3(16)$ & NS \\
\hline Diabetes mellitas & $1(5)$ & $2(4)$ & 0 & NS \\
\hline Pregnancy associated hypertension & $8(39)$ & $17(33)$ & 0 & $\mathrm{~b}=0.05$ \\
\hline
\end{tabular}

Data are presented as mean \pm standard deviation for numerical and number (\%) for categorical variables. MWL, maximum weight loss; NS, not significant. For P-values: $\mathrm{a}=$ low MWL $v$ s. medium MWL, b=low MWL $v$. high MWL, c=medium MWL vs. high MWL.

Table 2. Complications and outcomes of infants in the three groups.

\begin{tabular}{|c|c|c|c|c|}
\hline Variables & Low MWL $(n=21)$ & Moderate MWL $(n=52)$ & High MWL ( $n=18)$ & $\mathbf{P}$ \\
\hline Total days on oxygen (d) & $43 \pm 26$ & $61 \pm 33$ & $68 \pm 33$ & $a=0.06, b=0.03$ \\
\hline Oxygen dependence on DOL 28 & $10(56)$ & $44(85)$ & $18(100)$ & $\mathrm{b}=0.01$ \\
\hline PDA & $5(23)$ & $20(38)$ & $6(33)$ & NS \\
\hline IVH (any grade) & $4(19)$ & $11(21)$ & $3(17)$ & NS \\
\hline Periventricular leukomalacia & $2(9.5)$ & $2(4)$ & $2(11)$ & NS \\
\hline Pulmonary interstitial emphysema & $3(14)$ & $8(15)$ & $5(28)$ & NS \\
\hline Retinopathy of prematurity (any stage) & $16(76)$ & $42(82)$ & $15(83)$ & NS \\
\hline Inotropes use & $6(28)$ & $16(30)$ & $5(28)$ & NS \\
\hline Length of stay (d) & $80 \pm 26$ & $82 \pm 23$ & $86 \pm 24$ & NS \\
\hline
\end{tabular}

Data are presented as mean \pm standard deviation and number (\%). MWL, maximum weight loss; NS, not significant. For P-values, a=low MWL vs. medium MWL, b=low MWL vs. high MWL, c=medium MWL vs. high MWL. 
water volume, although rapid changes in serum osmolality might cause some intracellular water loss as well in ELBW infants. $2,7,8$ The ECW continues to diminish inversely during intrauterine life with maturation and constitutes about $60-65 \%$ of total body water at 6 months of gestational age compared to $45 \%$ at term. ${ }^{9}$ The mean maximum weight loss in ELBW infants in this report was $15.03 \%$, which is comparable to the difference between the fetal ECW volumes at gestational ages of 6 months and term. This finding may be pertinent and its patho-physiological basis could provide additional insight into the postnatal body water volumes and distribution during transition in ELBW infants. Premature birth may significantly modify body water metabolism and the dynamics of inter compartmental water flux might differ between fetal and postnatal lives of prematurely born neonates of comparable adjusted gestational age.

EPWL is physiologically regulated by prolactin in-utero and by atrial natriuretic factor post-natally.2,10,11 In addition to urinary output, which is inadequately controlled by immature renal functions, body water is insensibly lost via transcutaneous and pulmonary mucosal routes. These features are maximally exaggerated in ELBW infants secondary to several factors, such as, a thin cutaneous epidermal barrier; proportionately large exposed body surface area; stressful external ambience vis-a-vis artificial heating and phototherapy; high total body water content; a poikilothermic status and tachypnea. ${ }^{12,13}$ A lack of association of total or fractionated maximum EPWL with morbidities related to body water balance despite all these adverse conditions suggest the physiological competence, however limited, of ELBW infants, as well as, the appropriateness of neonatal fluid management. The wide range of EPWL in them could be attributed to the significant variability in the occurrences and severity of con-current morbidities, as well as to the maternal factors, severity of prematurity, degree of neonatal skin cornification, receipt of phototherapy, intensity of external heating and humidification etc. Antenatal steroid is reported to enhance renal maturation and reduce postnatal weight loss, although its receipt was not significantly different between the groups in our cohort. 4,14 We found maternal hypertension to be associated with a reduction in EPWL volume in our cohort of ELBW infants. This is a so far undocumented finding and could be attributed potentially to the release of endogenous fetal steroids due to stress. Maternal hypertension has multiple known adverse effects in neonates, ${ }^{15}$ although its implication in the water metabolism of growing fetus or prematurely born neonates has not been well documented. The mechanism of this observation deserves to be further investigated in order to fully explore the pathophysiological effects of PAH on the maternal-fetal unit. Fluid therapy, aimed to maintain a normal intravascular volume in which intake and output are closely balanced, is adequate for most uncomplicated cases of mother with hypertension. ${ }^{16}$ There is a paucity of evidence-based guidelines concerning intravenous fluid administration in pre-eclampsia and it is not known if maternal fluid management during hypertension has any implications in the fetus. ${ }^{15,16}$

EPWL is a well-recognized clinical entity common to all neonates. It is observed during the first few days of life and assumed to be physiological. Yet, limited normative data are available, even for healthy term newborn infants, and its physiological, as well as, pathological aspects are not well investigated. Term neonatal weight loss is documented to be brief with few children losing more than $10 \%$ below birth weight after 5 days, and a sustained loss of more than $10 \%$ of birth weight is unusual, although not commonly associated with underlying pathology. ${ }^{17}$ A systematic review, which included primary research studies with weight loss data for healthy, full-term, exclusively breastfed neonates during the first 2 weeks of life, found $7 \%$ to be the maximum allowable weight loss and suggested further research to understand the causes and implications of neonatal weight loss in their morbidity and mortality. ${ }^{18}$ EPWL can be evaluating as the maximum that a neonate can safely lose from birth weight, or as the pattern and limits of daily weight losses until recovery of birth weight. The day of life of maximum weight loss and the duration and day of life of recovery of birth weight also constitute important clinical aspects of a normal EPWL. 18 In order to determine EPWL to be pathological, the normal weight loss limits needs to be first defined via observational studies.

Evaluation of EPWL is more complicated in prematurely born neonates due to the physiological immaturity of their organ systems and consequent pathologies. To categorize their early postnatal weight loss as physiological is both unscientific and unsubstantiated. Anchieta et al. reported a weight loss of 5.9 to $13.3 \%$ in $<2500 \mathrm{~g}$ neonates during the first 2-3 weeks of life. ${ }^{19}$ Our earlier study in $<1000 \mathrm{~g}$ infants documented maximum EPWL to be $14.2 \pm 5.4 \%$ of birth weight and unassociated with neonatal morbidities independent of gestational age. ${ }^{4}$ Low EPWL was associated with
BPD and death in two earlier studies, neither of which specified the volume, range, or whether it was estimated as mean, total or maximum.5,6 In another report, early postnatal daily weight changes were documented to be unassociated with neonatal morbidities, and potentially protective for PDA, IVH and Oxygen dependence on DOL 28 in ELBW infants. ${ }^{20}$ The present analysis extends the previous reports by documenting a non contribution of graded maximum transitional weight loss to major neonatal morbidities in extremely preterm neonates. Even though, technically, the reported volume can not be regarded as physiological in ELBW infants like their term counterparts, it may be reasonable to state that the early postnatal transitional weight changes are important clinical component of the expedient process of transition to extra-uterine life in the critically ill extremely low birth weight neonates and should be allowed in the documented range for better outcomes.

\section{References}

1. Shaffer S, Quimiro CL, Anderson JV, Hall RT. Postnatal weight changes in low birth weight infants. Pediatr 1987;5:702-5.

2. Modi N, Betremieux P, Midgley J, Hartnoll G. Postnatal weight loss and contraction of the extracellular compartment is triggered by atrial natriuretic peptide. Early Hum Dev 2000;59:2 01-8.

3. Verma RP, Shibli S, Fang H. Clinical profile of early postnatal body weight changes in the extremely low birth weight infants. J Neonat Perinat Med 2010;3:1-7.

4. Verma RP, Shibli S, Fang H, Komaroff E. Clinical determinants and utility of early postnatal maximumweight loss in fluid management of extremely low birth weight infants. Early Hum Dev 2009;85:59-64.

5. Oh W, Pointdexter B, Perritt R, et al. Association between fluid intake and weight loss during the first ten days of life and risk of bronchopulmonary dysplasia in extremely low birth weight infants. J Pediatr 2005;47:786-9.

6. Wadhawan R, Oh W, Perritt R, et al. Association between early postnatal weight loss and death or BPD in small and appropriate for gestational age extremely low-birth-weight infants. J Perinatol 2007;27:359-64.

7. Bauer K, Versmold H. postnatal weight 
loss in preterm neonates $<1500 \mathrm{~g}$ is due to isotonic dehydration of the extracellular volume. Acta Ped Scand 1989;360:37-42.

8. Sedin G. Fluid management of the extremely premature infants. In: Hansen TN, McIntosh N. (eds). Current topics in neonatology. London; WB Saunders: 1995. pp 50-66.

9. Brans YW. Body fluid compartments in neonates weighing 1000 gms or less. Clin Perinatol 1986;13:403-17.

10. Baumgart S. Costarino A. Water and electrolyte metabolism of the micropremie. Clin Perinatol 2000;27:131-46.

11. Coulter MD. Prolactin: a hormonal regulator of the neonatal tissue water reservoir. Peds Res 1983;17:665-8.

12. Oh W. Fluid and electrolyte manage- ment of very low birth weight infants. Pediatr Neonatol 2012;53:329e-33e.

13. Gaylord MS, Wright K, Lorch K, et al. Improved fluid management utilizing humidified incubators in extremely low birth weight infants. J Perinatol 2001;21:438e43.

14. Omar SA, DeCristofaro JD, Agarwal BI, La Gamma EF. Effects of prenatal steroids on water and sodium homeostasis in extremely low birth weight neonates. Pediatrics 1999;104:482-8.

15. Backes CA, Markham K, Moorehead P, et al. Maternal preeclampsia and neonatal outcomes. J Pregnancy 2011;2011: 214365.

16. Anthony J, Schoeman LK. Fluid management in pre-eclampsia. Obstet Med 2013;6:100-4.
17. Wright CM, Parkinson KN. Postnatal weight loss in term infants: what is normal and do growth charts allow for it? Arch Dis Child Fetal Neonatal 2004;89:F254-7.

18. Noel-Weiss JN, Courant G, Woodend AK. Physiological weight loss in the breastfed neonate: a systematic review. Open Med 2008;2:e11.

19. Anchieta LM, Xavier CC, Colosimo EA, Souza MF. Weight of preterm newborns during the first twelve weeks of life 2-3 weeks of life. Braz J Med Biol Res 2003;36:761-70.

20. Verma R, Shibly S, Fang H, Pollack S. Do early postnatal body weight changes contribute to neonatal morbidities in the extremely low birth weight infants. J Neonatal Perinatal Med 2015;8:113-8. 